\title{
What is the Role of Double-Balloon Endoscopy in Patients Presenting with Obscure Gastrointestinal Bleeding?
}

\author{
Jung Ho Kim and Kwang An Kwon \\ Department of Internal Medicine, Gachon University Gil Hospital, Incheon, Korea
}

See "Double-Balloon Endoscopy in Overt and Occult Small Bowel Bleeding: Results, Complications, and Correlation with Prior Videocapsule Endoscopy in a Tertiary Referral Center" by Carlijn Hermans, Arnold Stronkhorst, Annemarie Tjhie-Wensing, et al., on page 69-75.

Small bowel lesions were very difficult to diagnose up until the development of video capsule endoscopy (VCE). It had been regarded as a 'no man's area' in many cases. Although VCE was developed to observe the small bowel lesions, it is difficult to observe in detail the lesion desired by the operator. Moreover, using VCE, it is difficult to perform a biopsy or to treat small bowel lesions. In particular, it is difficult to cope with an emergency situation in patients with gastrointestinal bleeding. A double balloon endoscopy (DBE) has been developed in 2001 by Yamamoto et al, and it has been useful to diagnose and treat small bowel diseases. ${ }^{1} \mathrm{DBE}$ is advantageous over VCE with respect to the diagnostic and therapeutic capabilities, including tissue sampling, tattooing, hemostasis, endoscopic mucosal resection, balloon dilation, and retention material removal. ${ }^{2}$

Obscure gastrointestinal bleeding (OGIB) refers to a state in which unknown origin bleeding or iron deficiency anemia is repeated or persistent after a negative evaluation, including upper gastrointestinal endoscopy and colonoscopy. OGIB can be classified into overt OGIB and occult OGIB. It is important to distinguish between overt bleeding and occult bleeding,

Received: January 19, 2017 Accepted: January 21, 2017

Correspondence: Kwang An Kwon

Department of Internal Medicine, Gachon University Gil Hospital, 21 Namdong-daero 774 beon-gil, Namdong-gu, Incheon 21565, Korea

Tel: +82-2-32-460-3778, Fax: +82-2-32-460-3408, E-mail: toptom@gilhospital.com

(cc) This is an Open Access article distributed under the terms of the Creative Commons Attribution Non-Commercial License (http://creativecommons.org/ licenses/by-nc/3.0) which permits unrestricted non-commercial use, distribution, and reproduction in any medium, provided the original work is properly cited. because they show different clinical courses and different treatment strategies. OGIB is not an uncommon problem encountered by gastroenterologists, since this may account for $5 \%-10 \%$ of all patients with gastrointestinal bleeding. ${ }^{3}$ OGIB usually occurs from lesions in the small bowel. Angiodysplasia is the most common cause of small bowel bleeding, accounting for about $70 \%$ of cases. ${ }^{4}$

The introduction of VCE and DBE has led to a major advance in the diagnosis and treatment of OGIB. The diagnostic yield of VCE and DBE in OGIB has been reported variously, depending on the different definitions of positive findings as well as on the type of bleeding investigated. Several studies have compared the yield of VCE and DBE, but have shown inconsistent results due to the small sample size. ${ }^{5}$ Arakawa et al. reported that a comparison of the overall diagnostic yield between VCE (54\%) and DBE (64\%) was not significantly different. ${ }^{6}$ They insisted that a complementary combination between VCE and DBE was useful for the management of OGIB. ${ }^{6}$ In other words, these two procedures should be considered complimentary and not competitive in the diagnosis and treatment of OGIB. ${ }^{5}$ Shishido et al. compared VCE-based and DBE-based detections of small bowel lesions in patients with OGIB and showed that small bowel lesions were detected by VCE in $44.9 \%$ and by DBE in $53.4 \%(p=0.01)$; agreement between VCE and DBE findings was good $(\kappa=0.76)^{7}$

Several investigators compared the diagnostic yields according to the type of OGIB and the timing of the procedures. For patients with prior overt OGIB, the diagnostic yield was less than that for current overt OGIB, which decreased substan- 
tially with the passage of time. In a 2004 study by Pennazio et al. investigating 100 patients undergoing VCE, they showed that the diagnostic yield was $92 \%$ for overt OGIB, $44 \%$ for occult OGIB, $67 \%$ for patients with prior overt OGIB who were studied within 10-14 days, and 33\% at 3-4 weeks postbleeding events. ${ }^{8}$ In a 2010 study that included 200 patients with OGIB undergoing DBE, the diagnostic yield was $77 \%$ for overt OGIB, $67 \%$ for patients with occult OGIB, and 59\% for patients with prior overt OGIB. ${ }^{9}$

VCE is usually performed first before DBE for evaluating OGIB. However, initial DBE can be considered in cases with massive bleeding or when VCE is contraindicated. Prediction of culprit lesions using VCE before DBE is useful for selecting the direction of approach. Gay et al. suggested that if the ratio of time-to-reach the lesion at VCE to the time-to-reach the cecum was 0.75 , the oral side should be considered first to reach the lesion with DBE. ${ }^{10}$ When DBE was selected for either the oral- or the anal- side approach, the diagnosis rate of DBE was lower than that of VCE in OGIB (50\% vs. $62 \%$ ). When DBE was performed by an approach using both sides, the diagnosis rate of DBE was superior to VCE (88\% vs. $46 \%){ }^{11}$ A DBE should be approached to both the oral side and the anal side to examine the entire small bowel intestine.

Positive VCE results augmented the diagnostic yield of subsequent DBE in a meta-analysis involving 10 studies. The diagnostic yield of subsequent DBE after positive VCE results was $75.0 \%$, while that after negative VCE results was only $27.5 \% .{ }^{12,13}$ Therefore, it is supported to precede before DBE.

In this issue, Hermans et al. ${ }^{14}$ presented their experience with DBE procedure in overt and occult small bowel bleeding, focusing on the results and correlation with prior VCE. Small bowel insertion during DBE was performed in 214 patients in 285 procedures. In $205 \mathrm{DBE}$ procedures (in 146 patients), the indication for DBE was OGIB. DBE for OGIB was preceded by VCE in 134 procedures (65\%), which was performed in 125 patients. DBE procedures showed a positive finding (only small bowel lesions) in 64\%. These 134 VCE procedures showed a negative result in 7 procedures (6\%). The other procedures showed a positive result. In 89 out of $134 \mathrm{DBE}$ procedures (66\%), the small bowel findings corresponded with VCE small bowel findings. Of these 134 DBE procedures, 41 procedures were negative despite a positive VCE. The findings from the three VCE negative and DBE positive procedures were erosion, terminal ileitis, and aspecific erythema. The median time between VCE and DBE procedures was 111 days (range, 1-1091 days). The diagnostic agreement between VCE and DBE results was weak $(\kappa=0.02)$. They insisted that it still may be clinically relevant to perform VCE to DBE due to the time consuming and invasive character of DBE.

This study has several limitations to be considered. Ac- cording to the international conference on capsule endoscopy consensus on OGIB, it is recommended that VCE should be performed as soon as possible (less than 2 weeks) in the evaluation of OGIB patients. ${ }^{15}$ The diagnostic yield of VCE in OGIB varies in accordance with the procedure timing of examination. The current study did not investigate the timing of the VCE procedure. This would have affected the diagnostic yield of VCE, as well as the weak diagnostic agreement between VCE and DBE results. Moreover, the period between the VCE and DBE procedures was too long, averaging 111 days. This would have affected the agreement rate and the correlation coefficient between the VCE and DBE results. Despite the large sample sizes, this study was only a single center, retrospective study. There may be a selection bias of patients. A multi-center, prospective study for the role and cost-effectiveness of VCE, single balloon endoscopy, and DBE in OGIB is necessary.

Conflicts of Interest

The authors have no financial conflicts of interests.

\section{REFERENCES}

1. Yamamoto H, Sekine Y, Sato Y, et al. Total enteroscopy with a nonsurgical steerable double-balloon method. Gastrointest Endosc 2001;53:216220.

2. Gerson LB, Fidler JL, Cave DR, Leighton JA. ACG Clinical guideline: diagnosis and management of small bowel bleeding. Am J Gastroenterol 2015;110:1265-1287; quiz 1288.

3. Longstreth GF. Epidemiology and outcome of patients hospitalized with acute lower gastrointestinal hemorrhage: a population-based study. Am J Gastroenterol 1997;92:419-424.

4. Cellier C. Obscure gastrointestinal bleeding: role of videocapsule and double-balloon enteroscopy. Best Pract Res Clin Gastroenterol 2008;22:329-340.

5. Tae CH, Shim KN. Should capsule endoscopy be the first test for every obscure gastrointestinal bleeding? Clin Endosc 2014;47:409-414.

6. Arakawa D, Ohmiya N, Nakamura M, et al. Outcome after enteroscopy for patients with obscure GI bleeding: diagnostic comparison between double-balloon endoscopy and videocapsule endoscopy. Gastrointest Endosc 2009;69:866-874.

7. Shishido T, Oka S, Tanaka S, et al. Diagnostic yield of capsule endoscopy vs. double-balloon endoscopy for patients who have undergone total enteroscopy with obscure gastrointestinal bleeding. Hepatogastroenterology 2012;59:955-959.

8. Pennazio M, Santucci R, Rondonotti E, et al. Outcome of patients with obscure gastrointestinal bleeding after capsule endoscopy: report of 100 consecutive cases. Gastroenterology 2004;126:643-653.

9. Shinozaki S, Yamamoto H, Yano T, et al. Long-term outcome of patients with obscure gastrointestinal bleeding investigated by double-balloon endoscopy. Clin Gastroenterol Hepatol 2010;8:151-158.

10. Gay G, Delvaux M, Fassler I. Outcome of capsule endoscopy in determining indication and route for push-and-pull enteroscopy. Endoscopy 2006;38:49-58.

11. Chen X, Ran ZH, Tong JL. A meta-analysis of the yield of capsule endoscopy compared to double-balloon enteroscopy in patients with small bowel diseases. World J Gastroenterol 2007;13:4372-4378.

12. Teshima CW, Kuipers EJ, van Zanten SV, Mensink PB. Double balloon 


\section{C cuncalenosscopy}

enteroscopy and capsule endoscopy for obscure gastrointestinal bleeding: an updated meta-analysis. J Gastroenterol Hepatol 2011;26:796-801.

13. Min YW, Chang DK. The role of capsule endoscopy in patients with obscure gastrointestinal bleeding. Clin Endosc 2016;49:16-20.

14. Hermans C, Stronkhorst A, Tjhie-Wensing A, et al. Double-balloon endoscopy in overt and occult small bowel bleeding: results, complications, and correlation with prior videocapsule endoscopy in a tertiary referral center. Clin Endosc 2017;50:69-75.

15. Pennazio M, Eisen G, Goldfarb N; ICCE. ICCE consensus for obscure gastrointestinal bleeding. Endoscopy 2005;37:1046-1050. 